\title{
Ost- und westdeutsche Spracheinstellungen
}

\author{
Gerhard Stickel
}

Die sprachlichen Besonderheiten der alten Bundesrepublik und der DDR sind in einer Vielzahl linguistischer Publikationen beschrieben (Anm. 1 u. 2). Zu den beglückenden Erfahrungen nach dem Fall der Berliner Mauer und der Öffnung der innerdeutschen Grenze gehörte zunächst für viele Menschen, dass Ost- und Westdeutsche sich trotz vierzigjähriger Trennung anscheinend ohne besondere Probleme verstehen können.

Das Gefühl ungetrübter sprachlicher Gemeinsamkeit und Übereinstimmung schien sich aber schon wenige Jahre nach der rasch vollzogenen staatlichen Vereinigung wieder abgeschwächt zu haben. In dem Maße, in dem sich die großen wirtschaftlichen Probleme im ostdeutschen Alltag konkret auswirkten und auch die Konjunktur in den westdeutschen Bundesländërn nachließ, konnte man in Gesprächen und auch in der öffentlichen Kommunikation immer wieder Äußerungen beobachten wie Wir verstehen uns wohl doch nicht oder Wir sprechen eigentlich zwei Sprachen. Mit derartigen Außerungen wurden und werden vermutlich in vielen Fällen keine semantischen Differenzen benannt, dass also Ost- und Westdeutsche z. B. mit Wörtern wie planen, Markt, Eigentum, demokratisch, ökonomisch oder anderen verschiedene Bedeutungen ver- 
binden. Wie in manchen Freundschafts- und Ehekrisen geht es dabei letztlich weniger um Verstehensdefizite als um Verständigungsschwierigkeiten, also nicht um mangelndes sprachliches Verständnis, sondern um fehlendes Einverständnis beim Aushandeln von Standpunkten und Interessen. Du verstehst mich nicht meint ja in der Zweierkommunikation oft nur: "Du verstehst vermutlich, was ich meine, willst aber offensichtlich nicht das, was ich will".

Der Eindruck zunehmender ost-westdeutscher Verständigungsskepsis oder gar -verdrossenheit ließ sich aber nicht auf umfassende Untersuchungen stützen, sondern lediglich auf die Beobachtung einzelner Gespräche und Äußerungen in den Medien. Die Mauer in den Köpfen ist ja seit mehreren-Jahren-ein-kon_sämtlichen Medien eifrig gepflegter Topos. Im Winter $1997 / 98$ bot sich mir dann eine Gelegenheit, den wechselseitigen Sprachwahrnehmungen von West- und Ostdeutschen mit einer empirischen Untersuchung nachzugehen, und zwar im Rahmen einer ganz Deutschland umfassenden Repräsentativerhebung vón Spracheinstellungen und -meinungen (Anm. 3). Die Umfrage galt mehreren sprachlichen Themen. Über die Ergebnisse ist schon an anderer Stelle ausführlich berichtet worden (Stickel 1999, Stickel/Volz 1999). Hier will ich nur die Fragen und Antworten zum sprachlichen - OstWest-Verhältnis in Deutschland kurz vorstellen und erörtern.

Ziel der Fragen zu diesem Themenbereich war es vor allem zu ermitteln, inwieweit der in der öffentlichen Diskussion vermittelte Eindruck von zunehmenden sprachbedingten Verständigungsproblemen zwischen West- und Ostdeutschen den Meinungen und Einstellungen der Bevölkerung tatsächlich entspricht. Befragt wurden insgesamt 2000 deutschsprachige Erwachsene, und zwar je 
rund 1000 in Ost- und in Westdeutschland (Anm. 4). Mit einer Eröffnungsfrage zum sprachlichen Ost-West-Verhältnis wurde die gesamte Stichprobe in zwei Teilgruppen geteilt: in die Befragten, die überhaupt deutliche sprachliche Unterschiede zwischen den alten und den neuen Bundesländern sehen, und diejenigen, die solche Unterschiede nicht bemerken. Die Frage lautete:

Meinen Sie, dass es deutliche sprachliche Unterschiede zwischen den alten und den neuen Bundesländern gibt?

Antwortmöglichkeiten waren: ja, sehr viele, ja einige und nein, keine bemerkenswerten.

Tabelle 1 verzeichnet die Verteilung der Antworten in Prozent:

Tabelle 1

\begin{tabular}{lccc}
\hline sprachl. Ost-West-Unterschiede? (in \%) & West & Ost & zus. (gew.) \\
\hline ja, sehr viele & 17,9 & 10,8 & 16,4 \\
ja, einige & 40,5 & 40,1 & 40,4 \\
nein, keine bemerkenswerten & 41,7 & 49,1 & 43,2 \\
\hline
\end{tabular}

Erstaunlich ist mit $43,2 \%$ der große Anteil der negativen Antworten, d. h. der Anteil der Gefragten, die keine bemerkenswerten Sprachdifferenzen zwischen Ost und West sehen. Vergleichsweise klein ist mit zusammen $16,4 \%$ der Anteil derer, die sehr viele Unterschiede annehmen. Mehr Westdeutsche als Ostdeutsche sehen sehr viele Unterschiede (17,9 vs. $10,8 \%)$. Dagegen nehmen mehr Menschen in den ostdeutschen Bundesländern an, dass es keine bemerkenswerten sprachlichen Unterschiede gebe. Hiernach ist der Anteil der Menschen, die sprachlich nichts Trennendes bemerken, in den ostdeutschen Bundesländern sogar noch größer als in den westdeutschen. Die moderate Meinung, dass es einige Differenzen gibt, ist mit rund $40 \%$ unter West- und Ostdeutschen ziemlich gleich verbreitet. 
Die folgenden speziellen Fragen wurden nur noch an die Teilgruppe der Probanden gerichtet, die überhaupt sprachliche Unterschiede (,sehr viele“ oder „einige") bemerken, d. h. 56,8\% der gesamten Stichprobe. Die erste Frage an diese Gruppe war:

Erscheinen Ihnen diese sprachlichen Unterschiede zwischen Ostdeutschen und Westdeutschen größer als die sprachlichen Unterschiede zwischen Norddeutschen und Süddeutschen?

Hier die tabellarische Zusammenfassung der Antworten:

Tabelle 2 (ohne „keine bemerkenswerten“ aus Tab. 1)

\begin{tabular}{lccc}
\hline Ost-West vs. Nord-Süd in \% & West & Ost & zus. (gew.) \\
\hline ja, größer & 29,4 & 23,4 & 28,4 \\
nein, nicht größer & 70,6 & 76,6 & $(1,6)$ \\
\hline
\end{tabular}

Mehr als zwei Drittel der zu diesem Thema Befragten sehen demnach die sprachlichen Unterschiede zwischen den alten und den neuen Bundesländern als Erscheinungen regionaler Sprachvarianz ähnlich den großregionalen sprachlichen Besonderheiten von Nord- und Süddeutschen. Bezogen auf die gesamte Menge der Befragten, einschließlich derjenigen, die keine Sprachunterschiede sehen, halten also weniger als $15 \%$ die Unterschiede in ostwestlicher Dimension für größer als die in nord-südlicher Vergleichsrichtung.

Als zentrale Frage schloss sich an:

Meinen Sie, dass die Sprachunterschiede in Ost und West die Verständigung in Deutschland sehr stark, etwas oder gar nicht behindern?

Zur Verteilung der Antworten die folgende Tabelle: 
Tabelle 3 (ohne „keine bemerkenswerten“ aus Tab. 1)

\begin{tabular}{lccc}
\hline Sprachl. Verständigungshindernisse? in \% & West & Ost & zus. (gew.) \\
\hline sehr stark & 4,8 & $\mathbf{1 , 5}$ & 4,2 \\
etwas & 36,9 & 39,0 & 37,3 \\
gar nicht & $\mathbf{5 8 , 3}$ & $\mathbf{5 9 , 6}$ & 68,6 \\
\hline
\end{tabular}

Beachtenswert ist, dass-nur 4,2\% dieser Teilgruppe in-den ost-westdeutschen Sprachdifferenzen ein sehr starkes Verständigungshindernis sehen. Bezogen auf die gesamte Stichprobe, also unter Berücksichtigung auch der Gefragten, die keine deutlichen - pprachlichen Unterschiede sehen, sind dies nur etwa (2,49\%. Geradezu komplementär groß ist hierzu mit fast $60 \%(58,6 \%)$ der Anteil derjenigen der Teilgruppe, die keine sprachlich bedingten Verständigungshindernisse bemerken. $\mathrm{Da}$ auch hierzu noch alle diejenigen $\mathrm{zu}$ berücksichtigen sind, die überhaupt keine bemerkenswerten sprachlichen Ost-West-Unterschiede annehmen, können wir schließen, dass insgesamt gut drei Viertel $(76,4 \%)$ aller an der Umfrage Beteiligten meinen, es gebe keine sprachbedingten Verständigungsprobleme zwischen West- und Ostdeutschen. Die Vermutung einer seit einigen Jahren zunehmenden wechselseitigen Sprachskepsis, die auch ich hatte, wird demnach durch die Umfrageergebnisse deutlich widerlegt. Tabelle 3 zeigt, dass der kleine Anteil der Verständigungsskeptiker mit 4,8\% in Westdeutschland etwas größer ist als in Ostdeutschland $(1,5 \%)$. Ich hatte das umgekehrte Verhältnis vermutêt, eine Vermutung, die vielleicht durch das verbreitete Vorurteil von den ständig klagenden Ostdeutschen (,Jammerossis') mitverursacht war.

Anschließend wurden die Probanden, die sprachliche Unterschiede bemerken, nach Beispielen für typische Wörter und Wendungen aus dem Sprachgebrauch der Menschen im jeweils anderen Landesteil gefragt, also nach typischen ostdeutschen bzw, typisch westdeutschen Ausdrücken. Die Frage lautete: 
Fallen Ihnen typische Wörter und Wendungen in der Sprache von Menschen aus den (je nach Herkunft der Gefragten) neuen/alten Bundesländern auf? Wenn ja, nennen Sie bitte zwei davon.

Die Frage erwies sich für eine quantitative Betrachtung der Ergebnisse als wenig geeignet, zumal nur etwa ein Viertel der gefragten Westdeutschen $(24,9 \%)$ und knapp die Hälfte der gefragten Ostdeutschen $(46,7 \%)$ Beispiele angeben konnten oder wollten. Es ergaben sich dennoch zwei lange Beispiellisten, die aber wegen der vielen Einmalnennungen für eine statistische Auswertung unergiebig sind. Wiedergegeben werden im Folgenden nur die Anfänge der Häufigkeitslisten. Erfasst sind sowohl objektsprachliche Ausdrücke, also Beispielwörter und -wendungen, als auch metasprachliche Bezeichnungen von typischen sprachlichen Erscheinungen. Die Beispielwörter und -wendungen sind kursiv gesetzt.

Tabelle 4

\begin{tabular}{lr}
\hline Rroilech ostdeutsch" (aus westdeutscher Sicht) \\
\hline Sächsisch an sich/sächsischer Tonfall \\
ick/icke (für ich) \\
nu/nü/no (statt ja) \\
(Ein-/Zwei-/Vier-)raumwohnung (statt -zimmerwohnung) \\
Kaufhalte (statt Supermarkt) \\
Kallektiv & $\mathbf{1 1}$ \\
Raste (statt Plastik) & $\mathbf{5}$ \\
Zeitangaben (z. B. drei viertel vier) & 5 \\
\hline
\end{tabular}

Deutlich ist, dass bei diesen Angaben aus westdeutscher Sicht dialektale Besonderheiten des Sächsich-Thüringischen $(n u / n u ̈)$ und Berlinischen (ick/icke) mit überregionalen ostdeutschen Spezifika (Broiler, Einraumwohnung, Kollektiv, Plaste) durcheinander gehen. Die als typisch ostdeutsch erachtete Form von Zeitangaben (drei viertel vier statt viertel vor vier) beruht zudem auf einer Fehlein- 
schätzung. Die regionale Verteilung der unterschiedlichen Zeitangaben verläuft ja quer zu der (ehemaligen) ostwestdeutschen Grenze.

Es folgt der Anfang der Häufigkeitsliste der Beispiele für typisch westdeutschen Sprachgebrauch aus ostdeutscher Sicht.

Tabelle 5

\begin{tabular}{lc}
\hline typisch westdeutsch" (aus ostdeutscher Sicht) & \\
Supen & 22 \\
-Zeitangabe (z.B. viertel vor vier) & 18 \\
Ossi(s) & 9 \\
(Brat)Hähnchen (statt Broiler) & 9 \\
\hline Anglizismen/Amerikanismen/englische Wörter" & 8 \\
Schaun mer mal! & 7 \\
Cood & 6 \\
Grüß Gott & 6 \\
Kids & 6 \\
okay/o. k. & 6 \\
Shopping/Shoppen (statt Einkaufen) & 6 \\
Team (statt Kollektiv) & 6 \\
Flieger (statt Flugzeug) & 5 \\
außen vor lassen/bleiben. & 4 \\
\hline
\end{tabular}

Auch unter diesen Nennungen aus ostdeutscher Sicht finden sich Regionalismen wie Grüß Gott! und viertel vor vier neben überregionalen westdeutschen Spezifika wie dem häufigeren Anglizismengebrauch in den westlichen Bundesländern. Der Vergleich der Tabellen 4 und 5 macht unter anderem auf das Kuriosum aufmerksam, dass die $\mathrm{Be}$ zeichnungen für gebratenes Geflügel (Broiler vs. Brathähnchen) geradezu Schibboleth-Qualität für die sprachliche Ost-West-Wahrnehmung hat. Die Liste der Westspezifika aus" ostdeutscher Sicht (auch in der hier nicht wiedergegebenen Fortsetzung) ist übrigens merklich reichhaltiger als die Beispielliste aus umgekehrter Sicht. Wenngleich die Verständigungsskepsis in den ostdeutschen Ländern geringer ist, als ich vermutet hatte, ist offensichtlich die Auf- 
merksamkeit Ostdeutscher gegenüber dem westdeutschen Sprachgebrauch etwas größer als in der Gegenrichtung.

Die abschließende Frage zu den ost-westdeutschen Spracheinstellungen galt den Meinungen zur künftigen Entwicklung:

Was glauben Sie, wann werden sich West- und Ostdeutsche in ihrem Sprachverhalten einander angeglichen haben? Antwortmöglichkeiten waren: in den nächsten 10 Jahren, in mehreren Jahrzehnten und wahrscheinlich nie.

Tabelle 6 verzeichnet die Verteilung der Antworten:

Tabelle 6 (ohne „keine bemerkenswerten“ aus Tab. 1)

\begin{tabular}{lccc}
\hline Sprachliche Angleichung? (in \%) & West & Ost & zus. (gew.) \\
\hline in 10 Jahren & 24,5 & 34,5 & 26,6 \\
in mehreren Jahrzehnten & 25,2 & 29,6 & 26,0 \\
wahrscheinlich nie & 50,3 & 36,0 & \\
\hline
\end{tabular}

Der Anteil der Ostdeutschen, die eine sprachliche OstWest-Angleichung schon für das nächste Jahrzehnt annëhmen, ist mit 34,5\% merklich größer als der Anteil der Westdeutschen (24,5\%) mit dieser Meinung. Andererseits nimmt gut die Hälfte $(50,3 \%)$ der Westdeutschen an, also deutlich mehr als Ostdeutsche $(36,0 \%)$, dass es zu einer sprachlichen Angleichung wahrscheinlich nie kommen werde. Dieses leicht-asymmetrische Verhältnis erklärt sich zum Teil möglicherweise schon aus der unterschiedlichen Bevölkerungsgröße in Ost- und Westdeutschland. Eine Minderheitsgruppe ist im Allgemeinen eher an Angleichung interessiert als eine Mehrheitsgruppe.

Für wichtiger an den Antworten zu dieser Frage halte ich, dass nur eine Minderheit aus beiden deutschen Teilgebieten eine rasche sprachliche Angleichung für wahrscheinlich hält. Eine deutliche Mehrheit von zusammen $73,3 \%(26,0 \%+47,3 \%)$ erwartet eine sprachliche Anglei- 
chung von-West-und-Ostdeutschen erst nach mehreren Jahrzehnten oder schließt sie sogar ganz aus. Dies sollte -keineswegs als Zeichen einer vorherrschendèn innerdeutschen Sprach- und Verständigungsskepsis gesehen werden. Die Vermutung einer solchen Skepsis wurde ja durch einige der vorausgehenden Fragen und Antworten widerlegt, besonders durch die Antworten auf die Frage nach den sprachlichen Verständigungshindernissen (s. o. Tab. 3). Ich sehe in der Verteilung der Antworten auf die letzte Frage in erster Linie eine positive Einschätzung der Beständigkeit regionaler Sprachvarietäten in Deutschland.

Dass die regionalen Varietäten des Deutschen derzeit vorwiegend positiv bewertet werden, ergab im Rahmen der Erhebung eine andere Frage, die nicht auf Ost-WestEinstellungen abzielte (Anm. 5). Hierbei zeigte sich, dass fast zwei Drittel der Gefragten (60,6\%) regional geprägten Sprachgebrauch uneingeschränkt akzeptieren und nur 4,5\% ihn prinzipiell ablehnen. Die Meinungsverteilung wăr hierbei in den alten und neuen Bundesländern nahezu gleich. Vor dem Hintergrund dieses Ergebnisses, das die Toleranz und Offenheit der meisten Deutschen gegenüber regionalen Sprachvarietäten zu erkennen gibt, erscheinen die Antworten auf die Fragen nach der sprachlichen Angleichung von Ost- und Westdeutschen als unproblematisch. Die regionalen Besonderheiten der deutschen Sprache im Norden und Süden wie auch die im Osten und Westen werden offensichtlich von der Mehrzahl der Deutschen nicht als Nachteil, als Hindernis für die innerdeutsche Verständigung angesehen, sondern eher als Vorteil, als erhaltenswerter sprachlicher Reichtum, der nicht schon in wenigen Jahren durch völlige sprachliche Angleichung aufgegeben werden sollte.

- Als Ergänzung möchte ich noch die ost-west-spezifischen Antwortverteilungen zu zwei weiteren Fragen vor- 
stellen, bei denen es um die Ermittlung von Einstellungen zu anderen Sprachen geht; die in Kontakt zum Deutschen stehen. Zunächst das Verhältnis der deutschsprachigen Deutschen zu den durch Arbeitsmigration entstandenen Minderheitensprachen in Deutschland. Hierzu lautete eine Frage:

In Deutschland gibt es neben alten Minderheitensprachen wie.Sorbisch in der Lausitz oder Dänisch in Schleswig seit einigen Jahrzehnten auch Minderheitensprachen von $z . T$. großen Zuwanderergruppen, darunter Türkisch, Italienisch und Spanisch. Finden Sie diese Mehrsprachigkeit in Deutschland gut oder schlecht oder ist sie Ihnen egal?

Hierzu die Verteilung der Antworten in Tabelle 7:

Tabelle 7

\begin{tabular}{lccc}
\hline Mehrsprachigkeit in Deutschland (in \%) & West & Ost & zus. (gew.) \\
\hline gut & 25,6 & 25,0 & $(25,5)$ \\
schlecht & 17,7 & 15,5 & 17,2 \\
mir egal & 56,7 & 59,5 & 57,2 \\
\hline
\end{tabular}

Die Einstellungen zu den neuen Minderheitensprachen in Deutschland sind unter den befragten Ost- und Westdeutschen nahezu gleich verteilt. Bemerkenswerter als die geringe Ost-West-Differenz ist hier, dass nur ein Viertel aller Befragten zu der in Deutschland gegebenen Mehrsprachigkeit positiv eingestellt ist. Und ebenfalls bemerkenswert ist, dass die Mehrheit der Befragten in West und Ost den Zuwanderersprachen gleichgültig gegenübersteht. Der hohe Anteil der Gleichgültigen kompensiert die rund $17 \%$ der negativ Eingestellten eben nicht. Eine Zusatzfrage nach staatlicher Förderung der Minderheitensprachen ergab, dass die meisten der "Gleichgültigen" in West und Ost eine solche Förderung ablehnen, also zu den latenten Gegnern der innerdeutschen Mehrsprachigkeit gehören (Stickel/Volz, 38f.). 
Eine nahezu gleiche Meinungsverteilung unter Westund Ostdeutschen ergab auch eine Frage zur politischen Stellung der deutschen Sprache in der Europäischen Union. Die Frage war:

In der Europäischen Union gibt es 11 Amtssprachen, darunter Deutsch. Der alltägliche amtliche Sprachverkehr in den europäischen Behörden erfolgt jedoch weitgehend in den zwei so genannten Arbeitssprachen Englisch und Französisch. Von manchen deutschen Politikern, aber auch von Vertretern anderer Staaten wird gefordert, in den europäischen Behörden Deutsch als dritte Amtssprache neben Englisch und Französisch zu benutzen. Finden Sie diese Forderung gut?

Als Antwortmöglichkeiten wurden neben ja, finde ich gut und nein,"finde ich nicht gut auch bin unentschieden angeboten. Hier das Ergebnis:

Tabelle 8:

\begin{tabular}{lccc}
\hline EU-Arbeitssprache Deutsch? (in \%) & West & Ost & gege(gew.) \\
\hline Ja/gut & 54,6 & $55 ; 9$ & 55, \\
Nein, nicht gut & 16,0 & 15,0 & 15,8 \\
Bin unentschieden & 29,4 & 29,1 & 29,1 \\
\hline
\end{tabular}

Auch zu dieser Frage verhalten-sich-Ost-und-Westdeutsche nahezu gleichartig. Bemerkenswert ist auch hier der relativ hohe Anteil von fast 30\% derjenigen, die sich (noch) keine Meinung gebildet haben.

Der Teil der Umfrage, der sich auf die west-ostdeutschen Spracheinstellungen bezog, ging von der Annahme aus, die wechselseitige sprachliche Wahrnehmung und Bewertung sei von zunehmender Skepsis und Verständigungspessimismus geprägt. Diese Annahme wurde widerlegt. Stattdessen war den Antworten zu entnehmen, dass die meisten Menschen in den ehemals getrennten deut- 
schen Gebieten die ost-westlichen Sprachdifferenzen, sofern sie diese überhaupt bemerken, kaum anders sehen als die Unterschiede zwischen dem Sprachgebrauch in Nord- und Süddeutschland. Soweit regionale sprachliche Spezifika wahrgenommen werden, ist sie von der überwiegenden Mehrheit aller Befragten, die eine recht zuverlässige repräsentative Auswahl aus der Gesamtmenge aller erwachsenen Deutschen darstellen, positiv eingeschätzt worden.

Dass derzeit Westdeutsch und Ostdeutsch als zwei ausgeprägte, relativ beständige großregionale Varietäten der deutschen Sprache wahrgenommen werden, ist durch die Umfrage nicht bestätigt worden. Die Mehrzahl der Befragten lässt in den mitgeteilten Einstellungen und Meinungen keine markante Identifikation als Ostdeutsche bzw. Westdeutsche erkennen. Mit Ausnahme weniger „DDRismen" wie dem Broiler werden als Merkmale sprachlicher Varianz eher die Eigenschaften herkömmlicher Dialekte und regionaler Umgangssprachen wahrgenommen. Als Linguist kann man keine verlässlichen Prognosen zur Sprachentwicklung abgeben. Vielleicht erlauben Sie mir aber, so ganz nebenbei die Vermutung zu äußern, dass es für die sprachliche Wahrnehmung von Menschen in Deutschland schon bald wichtiger sein wird, ob sie als Sachsen oder Bayern, als Mecklenburger oder Schwaben identifiziert werden können denn als Ossis oder Wessis.

Kein signifikanter Unterschied ergab sich zwischen den Antwortverteilungen in Ost- und Westdeutschland bei Fragen nach der Einstellung zu den Minderheitensprachen in Deutschland und zur politischen Stellung der deutschen Sprache in der Europäischen Union, beides Fragen, die weniger die Vergangenheit als die sprachliche Gegenwart und Zukunft betreffen. Die Gleichartigkeit der Einstellungen lässt gesamtdeutsche Gemeinsamkeiten 
erkennen--leider-aber-nicht-nur im Positiven. Die gesamtdeutsche Gleichverteilung der an solchen sprachpolitischen Fragen Desinteressierten und Meinungslosen macht deutlich, dass es sprachliche Themen und Aufgaben in Deutschland gibt, die wichtiger sind als die schon zu lange diskutierten und von den Medien gern immer wieder aufgewärmten Fragen nach den ost-westdeutschen Sprachunterschieden. Die in Zeitungen und Fernsehen beharrlich suggerierte Mauer in den Köpfen entspricht nicht der tatsächlichen Sprachbefindlichkeit der Ost- und Westdeutschen und behindert die offentliche Diskussion zukunftsweisender Themen, nämlich der innerdeutschen Mehrsprachigkeit und der weiteren Entwicklung der deutschen Sprache in einem hoffentlich auch künftig mehrsprachigen Europa.

\section{Anmerkungen}

1. Eine erweiterte Fassung zu Spracheinstellungen erschien von Stickel unter dem Titel „Was West- und Ostdeutsche sprachlich voneinander halten“. In: Reiher, Ruth/Baumann, Antje (Hrsg.), Mit gespaltener Zunge, Berlin 2000 .

2. Siehe hierzu die Literaturverzeichnisse in Fleischer (1986) und Schlosser (1990). Zum Wechsel in der Bewertung der sprachlichen Unterschiede als Sprachvarianz oder als Sprachspaltung siehe besonders Hellmann (1989).

3. Die Erhebung wurde von der Fritz Thyssen Stiftung finanziell ermöglicht und von der GFM-GETAS (Hamburg) durchgeführt.

4. Die genauen Zahlen sind: 969 Personen in den westlichen, 1056 in den östlichen Bundesländern, zusammen also 2025 Personen. Die "krummen" Zahlen ergeben sich durch das Aussondern unvollständig und falsch ausgefüllter Fragebögen.

5. Näheres in Stickel (1999), Kap. 3.5. 
Literatur

Baker, Colin (1992): Attitudes and Language. Clavendon: Multilingual Matters.

Fleischer, Wolfgang (1986): Wortschatz der deutschen Sprache in der DDR. Fragen seines Aufbaus und seiner Verwendungsweise. Leipzig.

Giles, Howard, et. al. (1987): Research on Language Attitudes. In. U. Ammon/N. Dittmar/K. J. Mattheier (Hrsg.), Sociolinguistics. Soziolinguistik. 1. Vol. (HSK 3.1), Berlin/New York, S. 585-597.

Hellmann, Manfred W. (1989): Die doppelte Wende. Zur Verbindung von Sprache, Sprachwissenschaft und zeitgebundener politischer Bewertung am Beispiel deutsch-deutscher Sprachdifferenzierung. In: Josef Klein (Hrsg:), Politische Semantik. Bedeutungsanalytische und sprachkritische Beiträgé zur politischen Sprachverwendung. Opladen, S. 297-326.

Schlieben-Lange, Brigitte (1991): Soziolinguistik. 3. Aufl., Stuttgart/ Berlin/Köln.

Schlosser, Horst (1990): Die deutsche Sprache in der DDR zwischen Stalinismus und Demokratie. Historische, politische und kommunikative Bedingungen. Köln.

Stickel, Gerhard (1999a): Zur Sprachbefindlichkeit der Deutschen Erste Ergebnisse einer Repräsentativumfrage. In: G. Stickel (Hrsg.), Sprache - Sprachwissenschaft - Öffentlichkeit. Jahrbuch 1998 des Instituts für deutsche Sprache. Berlin/New York.

Stickel, Gerhard/Volz, Norbert (1999): Meinungen und Einstellungen zur deutschen Gegenwartssprache. Ergebnisse einer bundesweiten Repräsentativerhebung. amades Nr. 2/99, Mannheim. 